\title{
Cognitive Health Assessment and Establishment of a Virtual Cohort of Dementia Caregivers
}

\author{
Corinna Lathan ${ }^{a}$ Angela S. Wallace ${ }^{a}$ Rita Shewbridge ${ }^{a} \quad N^{a c o l e ~} \mathrm{Ng}^{b}$ \\ Glenn Morrison ${ }^{b}$ Helaine E. Resnick ${ }^{a}$ \\ ${ }^{a}$ AnthroTronix Inc., Silver Spring, Md., and ${ }^{b}$ Lumos Labs, San Francisco, Calif., USA
}

Key Words

Cognitive performance $\cdot$ Caregivers $\cdot$ Psychosocial factors $\cdot$ Dementia $\cdot$ Virtual cohort

\begin{abstract}
Background: Many factors impact caregivers' cognitive health and, by extension, their ability to provide care. This study examined the relationship between psychosocial factors and cognitive performance among dementia caregivers and established a virtual cohort of caregivers for future research. Methods: Data on 527 caregivers were collected via a Web-based survey that assessed cognitive performance. Caregiver data were compared to corresponding data from 527 age-, race-, gender-, and education-matched controls from a normative database. Caregiver self-reported sleep, stress, health, and social support were also assessed. Results: Caregivers performed significantly worse than controls on 3 of 5 cognitive subtests. Stress, sleep, perceived support, self-rated health, years of caregiving, race, and gender were significant predictors of cognitive performance. Conclusion: In this sample of dementia caregivers, psychosocial factors interacted in complex ways to impact cognitive performance. Further investigation is needed to better understand how these factors affect cognitive performance among caregivers. This could be accomplished by the establishment of a virtual cohort that facilitates the development of digital tools to support the evaluation and management of caregiver needs in a manner that helps them remain effective in their caregiving roles.
\end{abstract}


Lathan et al.: Cognitive Health Assessment and Establishment of a Virtual Cohort of Dementia Caregivers

\section{Introduction}

In 2014, more than 15 million family members and other unpaid caregivers provided care to people with Alzheimer's disease and other dementias in the USA [1]. Approximately two thirds of informal caregivers are women, $34 \%$ are aged 65 years or older [1], and many experience cognitive decline that can impact their ability to care for themselves and the people who depend on them [2]. The economics of informal caregiving are staggering: a recent report indicated that the value of informal eldercare in the USA is USD 522 billion annually, and USD 218 billion of that figure is accounted for by dementia caregiving [1, 3]. Because the cost of replacing informal care with formal, paid support is so high, research focused on understanding threats to effective caregiving has clear economic and policy implications.

Caregivers face many obstacles as they struggle to balance care provision with their other responsibilities such as caring for their own children, career development, and community engagement. Caregivers of persons with Alzheimer's disease are also exposed to numerous long-term stressors that stem from care provision itself. These include the demands supporting activities of daily living (e.g., eating, hygiene), aiding with higher-level tasks (e.g., talking, writing), and contending with fluctuations in mood (e.g., depression, anger) and behavior (e.g., agitation, paranoia) [4]. Not only has it been demonstrated that caregivers of Alzheimer's patients are at increased risk for impaired cognition, depression, anxiety, absenteeism, and increased health care utilization (e.g., physician visits, emergency room usage, and hospitalization), but also, as the severity of Alzheimer's symptoms increase, the mental and physical health outcomes of their caregivers decrease $[5,6]$.

The stress associated with caregiving negatively impacts cognitive functioning [4, 7-9]. It has also been shown that cognitive decline occurs as the result of stress and depression, affecting speed, attention, and executive function [10]. Gaugler et al. [11] showed that in-home caregivers of a relative had significant declines in cognitive performance after 3 years of caregiving. Vitaliano et al. [12] reported that people who cared for a spouse with Alzheimer's disease had a slower cognitive processing speed than non-caregivers. The latter results mirror a report from Caswell et al. [8] that showed caregivers of spouses with Alzheimer's disease have lower levels of complex attention and speed of information processing than noncaregivers. Recently, Corrêa et al. [13] demonstrated that caregivers exhibit significantly worse performance on information processing speed and working memory than healthy controls.

Not only does this growing body of research suggest a relationship between caregiving and cognitive decline [12], it also points to the idea that the effect of caregiving on cognitive function may depend in part on the degree to which caregivers experience psychosocial risk factors. Poor cognitive function among dementia caregivers may be mediated by depressed mood [12], distress [8], hostility, metabolic factors [4], and the quality and quantity of sleep $[4,8]$. A better understanding of how psychosocial factors impact the relationship between caregiving and cognition could help inform the design of programs and guide therapies to address these detrimental effects, thereby helping caregivers to maintain their important role [10].

Given previously observed relationships between chronic stress, psychophysiological functioning, and cognitive factors, we established the Health-eBrain Study (www.HealtheBrainStudy.org). This is the first large, Web-based study of informal dementia caregivers. The purpose of this report is to describe the Health-eBrain cohort and report the relationship between caregivers' psychosocial factors and their cognitive performance. We recruited dementia caregivers to the study website and administered both a lifestyle survey and a Web-based cognitive assessment. These protocols allowed us to assess caregivers' demo- 
graphic and psychosocial profiles, and to examine relationships between these factors and cognitive performance. Our working hypothesis was that caregivers would exhibit more cognitive impairment than matched controls, and that among caregivers, psychosocial and lifestyle factors such as sleep and stress would have mitigating effects on cognitive performance.

\section{Methods}

This study was approved by the AnthroTronix Institutional Review Board.

Participant Recruitment

This cross-sectional study targeted caregivers of people with dementia. Caregivers were recruited through nonprofit foundation networks including the BrightFocus ${ }^{\circledR}$ Foundation and the Geoffrey Beene Foundation Alzheimer's Initiative, as well as through an invitation to Lumos Labs' database. Participants were directed to the study website (www.Health-eBrainStudy.org). Eligible respondents were 18 years or older and caring for someone with dementia at the time the respondent (caregiver) completed the online survey. Consent was obtained with an online form, which was followed by administration of the lifestyle survey. Once the lifestyle survey was completed, caregivers were offered a second online consent form for Lumos Labs' Brain Performance Test (BPT), which consists of 5 subtests. After administration of the BPT, caregivers were given the option to remain in the Health-eBrain Study registry and to be contacted for future studies. Five hundred and twenty-seven caregivers completed the lifestyle survey and the 5 subtests of the BPT, and 378 opted to remain part of the registry for future studies.

\section{Lifestyle Survey}

Caregivers were given a survey that collected information on demographics, duration of caregiving, and perceptions concerning stress and the availability of social support. Demographic information included age, gender, race, and education (some high school, high school graduate, some college, associate's degree, bachelor's degree, or graduate or professional degree). Duration of caregiving was assessed with the question: 'How many years have you been providing care?' Caregivers' self-reported stress level was assessed with the question: 'On a scale of 1 to 5 with 1 being "no stress," and 5 being "a great deal of stress," how much stress would you say you have in your life?' Self-reported sleep was assessed with the question: 'How much sleep do you typically get each night?' The following response options were offered: 4, 5, 6, 7, 8, 9, or $10 \mathrm{~h}$. To assess social support, caregivers were asked: 'On a scale of 1 to 7 with 1 being "not supported at all" and 7 being "extremely supported," how supported do you feel in your personal life?' Finally, caregivers were asked to rate their overall health by answering the question 'How would you describe your health?' with the following response options: 'poor', 'fair', 'very good', and 'excellent'.

\section{Cognitive Assessment}

The BPT is a Web-based battery of cognitive assessments that measures function across several domains. The BPT was designed to replicate pencil-paper tests (where applicable) for which a shift to computerized administration does not negatively impact the test mechanics. BPT results are consistent with corresponding pencil-paper testing [14-16]; the test is repeatable, changes predictably with age, is highly intercorrelated, and captures multiple, distinct cognitive abilities $[14,16,17]$.

The BPT is optimized for online administration in an unsupervised environment, and was therefore well suited to this research environment. The BPT battery used in this study 
included 5 subtests: Digit Symbol Coding, Forward Memory Span, Reverse Memory Span, Trail Making A, and Trail Making B. The battery took between 20 and 30 min to complete.

Digit Symbol Coding. This subtest is similar to the Wechsler Adult Intelligence Scale's Digit Symbol Substitution Task. This test measures information processing speed and relies heavily on visual search and memory [18]. The subtest lasts $90 \mathrm{~s}$, and caregivers used the keyboard to match a series of numbers that correspond to randomly generated symbols as quickly as possible. The primary measure is the number of correct responses minus the number of incorrect responses.

Forward and Reverse Memory Span. These subtests are derived from the Corsi blocktapping test [19], which assesses visuospatial short-term working memory. Forward Memory Span assesses the storage and maintenance components of working memory, whereas Reverse Memory Span evaluates information storage and information processing [20]. In computerized versions of these tasks, circles flash on the screen and caregivers reproduce the sequences by clicking on the circles [21,22]. The subtest concludes when 2 consecutive errors on 1 sequence length are made. The primary measure is 'numbers correct'.

Trail Making $A$ and $B$. Trail Making A involves connecting 25 encircled numbers that are randomly distributed across the computer screen by clicking on them in sequence. Task requirements are similar for Trail Making $B$, except that the participant alternates between numbers and letters (e.g., 1, A, 2, B, 3, C, etc.). Trail Making provides information on attention, visual search, scanning, processing speed, mental flexibility, and executive functions. Originally part of the 1944 Army Individual Test Battery [23], Trail Making was later incorporated into the Halstead-Reitan Neuropsychological Battery [24]. For both Trail Making A and Trail Making B, the primary measure is 'completion time' (there is no time limit).

Each BPT subtest included clear, interactive tutorials for which respondents were required to demonstrate a minimum level of proficiency prior to being presented with the task. The cycle of tutorial/subtest was repeated until all subtests were completed. All BPT subtest scores are scaled following a percentile rank-based inverse normal transformation, a protocol used in widely accepted measures of cognitive function such as the Wechsler Adult Intelligence Scale [25]. Normative tables are created for each BPT subtest, and the raw score from each subtest (e.g., the number of correct responses, completion time, etc.) is ranked to obtain a percentile for each raw score. The position of that percentile on a normal distribution is used to convert the raw score into a scaled score where the distribution has a mean of 100 and an SD of 15.

\section{Data Analysis}

To assess differences in cognitive performance between caregivers and controls, each caregiver was matched on age, gender, race, and education with a unique control from Lumos Labs' reference database of individuals who completed the same BPT battery, for a total of 527 controls and 527 caregivers. For each BPT subtest, two-sample t tests were used to assess differences in mean responses between caregivers and controls. Subtests on which we observed significant differences in performance between caregivers and controls were examined further in regression analyses. Stepwise linear regression was used to explore associations between psychosocial factors and cognitive performance among the dementia caregivers. We ran separate models for each relevant BPT subtest outcome. In each set of models, gender, race, education, years of caring, stress, hours of sleep per night, perceived social support, and perceived health were examined as independent variables. We specified an $\alpha$ criterion of $\leq 0.09$ for entry into the models. The regression analyses could only be completed for the caregiver data, as we did not have psychosocial survey data for the controls obtained from the deidentified database. 
Dementia

and Geriatric

Cognitive Disorders

Table 1. Demographics of the Health-eBrain Study virtual cohort $(n=527)$
Table 2. BPT t test results for matched controls versus caregivers $(n=527)$

\begin{tabular}{|c|c|c|c|}
\hline \multicolumn{4}{|c|}{ Dement Geriatr Cogn Disord Extra 2016;6:98-107 } \\
\hline DOI: $10.1159 / 000444390$ & \multicolumn{3}{|c|}{$\begin{array}{l}\text { (c) } 2016 \text { The Author(s). Published by S. Karger AG, Basel } \\
\text { www.karger.com/dee }\end{array}$} \\
\hline \multicolumn{4}{|c|}{$\begin{array}{l}\text { Lathan et al.: Cognitive Health Assessment and Establishment of a Virtual Cohort of } \\
\text { Dementia Caregivers }\end{array}$} \\
\hline \multicolumn{4}{|l|}{ Gender } \\
\hline Female & & 464 & $88.0 \%$ \\
\hline Male & & 63 & $12.0 \%$ \\
\hline \multicolumn{4}{|l|}{ Race } \\
\hline White & & 497 & $94.3 \%$ \\
\hline Non-White & & 37 & $5.7 \%$ \\
\hline \multicolumn{4}{|l|}{ Education } \\
\hline Some high school & & 10 & $1.9 \%$ \\
\hline High school graduate & & 51 & $9.7 \%$ \\
\hline Some college & & 115 & $21.8 \%$ \\
\hline Associate's degree & & 62 & $11.8 \%$ \\
\hline Bachelor's degree & & 129 & $24.5 \%$ \\
\hline Graduate or profession & nal degree & 160 & $30.4 \%$ \\
\hline \multicolumn{4}{|c|}{ Employment } \\
\hline Employed & & 254 & $48.2 \%$ \\
\hline Unemployed & & 272 & $51.6 \%$ \\
\hline \multicolumn{4}{|c|}{ Relationship to care recipient } \\
\hline Family member & & 270 & $51.2 \%$ \\
\hline Spouse/significant othe & er & 200 & $38.0 \%$ \\
\hline Paid caregiver & & 42 & $8.0 \%$ \\
\hline In-law & & 6 & $1.1 \%$ \\
\hline Friend & & 5 & $0.9 \%$ \\
\hline Other & & 4 & $0.8 \%$ \\
\hline
\end{tabular}

\begin{tabular}{lrrc}
\hline & Caregivers & Controls & \multicolumn{1}{l}{$\mathrm{t}$} \\
\hline Digit Symbol Coding & $95.51(18.96)$ & $102.00(15.14)$ & $6.13^{* * *}$ \\
Forward Memory Span & $97.69(16.56)$ & $102.71(14.30)$ & $5.27^{* * *}$ \\
Reverse Memory Span & $99.20(17.39)$ & $103.28(14.91)$ & $4.08^{* * *}$ \\
Trail Making A & $100.70(18.14)$ & $99.46(14.70)$ & -1.21 \\
Trail Making B $^{\text {a }}$ & $98.70(16.83)$ & $100.55(14.66)$ & $1.90^{\text {a }}$ \\
\hline
\end{tabular}

Values are given as means (SD). Caregivers and controls were matched for age, gender, race, and education. ${ }^{\mathrm{a}} \mathrm{p}<0.10$; *** $\mathrm{p}<0.001$.

\section{Results}

Data were collected from 527 dementia caregivers. These caregivers had an average age of 60.1 years, the vast majority (88\%) was female, and over half were employed at the time of the study (table 1). Most respondents reported caring for a loved one (family member: $51.2 \%$; spouse/significant other: $38.0 \%$ ), and nearly $60 \%$ of the care recipients had a diagnosis of Alzheimer's disease.

Table 2 shows that the caregivers' cognitive performance was significantly lower than that of the matched controls on 3 of the 5 subtests of the BPT: Digit Symbol Coding, Forward Memory Span, and Reverse Memory Span ( $<<0.001)$. These tests were the focus of our regression analyses among the dementia caregivers. The results of these analyses for each BPT subtest are shown in table 3. These results show adjusted effects of individual variables as well as variables with interaction effects. Only variables that were significant at $p<0.05$ are presented. It is important to note that despite the statistical significance of the predictor variables shown in the table, the regression models in table 3 explained less than $7 \%$ of the variance for all 3 subtests. 
Table 3. Regression results $(n=527)$

\begin{tabular}{|c|c|c|c|c|c|c|}
\hline & \multicolumn{2}{|c|}{ Digit Symbol Coding } & \multicolumn{2}{|c|}{ Forward Memory Span } & \multicolumn{2}{|c|}{ Reverse Memory Span } \\
\hline & $\beta(\mathrm{SE})$ & $\mathrm{t}$ & $\beta(\mathrm{SE})$ & $\mathrm{t}$ & $\beta(\mathrm{SE})$ & $\mathrm{t}$ \\
\hline Race (non-White vs. White) ${ }^{a}$ & & & & & $-5.9(3.0)$ & $-2.0^{*}$ \\
\hline Stress ${ }^{b}$ & $10.7(5.4)$ & $2.0^{*}$ & & & $-5.8(2.9)$ & $-2.0^{*}$ \\
\hline Hours of sleep ${ }^{c}$ & $10.5(3.2)$ & $3.3^{* *}$ & $3.5(1.4)$ & $2.6^{*}$ & & \\
\hline Years of caring ${ }^{\mathrm{d}}$ & & & $1.3(0.6)$ & $2.3^{*}$ & & \\
\hline Perceived support $\mathrm{e}^{\mathrm{e}}$ & & & $8.3(2.6)$ & $3.2^{* *}$ & $-4.7(2.0)$ & $-2.3^{*}$ \\
\hline Stress $\times$ sleep & $-2.1(0.8)$ & $-2.6^{*}$ & & & & \\
\hline Sleep $\times$ support & & & $-0.8(0.3)$ & $-2.4^{*}$ & & \\
\hline Gender $^{\mathrm{f}} \times$ health $^{\mathrm{g}}$ & $11.6(4.1)$ & $2.8^{* *}$ & & & & \\
\hline Education $\times$ years & & & $-0.3(0.1)$ & $-2.3^{*}$ & & \\
\hline
\end{tabular}

${ }^{*} \mathrm{p}<0.05,{ }^{* *} \mathrm{p}<0.01{ }^{\mathrm{a}}$ White $=0$, non-White $=1{ }^{\mathrm{b}}{ }^{\mathrm{b}}$ No stress $=1$, great deal of stress $=5$ (mean $=3.67$, $\mathrm{SD}=0.87) .{ }^{\mathrm{c}} 4,5,6,7,8,9$, or $10 \mathrm{~h}($ mean $=6.48, \mathrm{SD}=1.23) .{ }^{\mathrm{d}} 1-41$ years $($ mean $=5.03, \mathrm{SD}=4.27) .{ }^{\mathrm{e}}$ Not supported at all $=1$, extremely supported $=7$ (mean $=3.83, \mathrm{SD}=1.69) .{ }^{\mathrm{f}}$ Male $=0$, female $=1 .{ }^{\mathrm{g}}$ Poor $=1$, fair $=2$, very good $=3$, excellent $=4($ mean $=2.81, \mathrm{SD}=0.66)$.

For Digit Symbol Coding, reported hours of sleep per night was a positive predictor of performance such that for each additional hour of reported sleep per night there was a 10.5point increase in performance. Similarly, increasing stress predicted performance on this test such that for each additional level of reported stress there was a 10.7-point increase in performance on Digit Symbol Coding. However, there was a significant interaction between sleep and stress such that above $5.5 \mathrm{~h}$ of sleep the effect of stress on performance was negative. The impact of self-rated health on Digit Symbol Coding performance depended on gender such that female caregivers scored 11.6 points higher on Digit Symbol Coding as the level of self-rated health increased.

Perceived support was a positive predictor of performance on Forward Memory Span such that for each increased level of reported support, there was an 8.3-point increase in performance. Similarly, each additional hour of reported sleep was associated with a 3.5-point increase in performance on this test. However, there was a small but significant interaction between sleep and perceived support such that as the number of hours of sleep and the level of perceived support both increased, test performance decreased by 0.8 points. Years of caregiving predicted performance such that for each additional year of caring there was a 1.3-point increase in Forward Memory Span performance; however, due to a small but significant interaction with education, as educational level and years of caregiving both increased, performance decreased by 0.3 points.

Non-White caregivers scored 5.9 points lower than Whites on Reverse Memory Span, and stress was inversely associated with performance on this test such that each increasing level of reported stress was associated with a 5.7-point decline in Reverse Memory Span performance. Perceived support had a negative impact on performance such that for each increasing level of perceived support there was a 4.7-point decline in Reverse Memory Span performance.

\section{Discussion}

When comparing cognitive performance between dementia caregivers and matched controls, our data are consistent with previous results showing that dementia caregivers perform worse than non-caregivers on tests of attention, information processing, processing 
speed, and short-term working memory $[8,9,12,13,26]$. Our results also support other studies showing that, in general, better psychosocial functioning such as with reduced stress improves cognitive performance [27]. Notably, our large sample permitted an examination of how psychosocial factors such as stress, sleep, perceived health, and perceived level of support - alone and in combination - have complex effects on multiple cognitive domains among dementia caregivers. Our data also show that among dementia caregivers, gender, race, education, and years as a caregiver are associated with various cognitive performance domains.

The public health implications of our findings are tied to the fact that poor cognitive performance among caregivers has the potential to negatively impact the availability and quality of informal care $[2,12]$. As caregivers of spouses with dementia are typically older themselves, they may experience difficulty performing complex activities of daily living for themselves as well as the recipient of their care. For example, caregivers typically oversee medication management, a complex task that is particularly challenging among older adults in whom polypharmacy is common [28]. Medication management requires understanding of interrelationships between multiple concepts such as dosing, whether medicines should be taken with food, and at what time of day they should be taken. These organizational demands are augmented by the fact that older adults often take multiple medications, these medications often resemble one another, pill bottles are easily lost, the writing is small, and prescription renewals are often managed by multiple care providers. When caregivers experience cognitive decline, they may be less capable of managing medications as well as myriad other tasks that are necessary to keep a care recipient at home rather than in a nursing home or other residential care facility [12]. Caregivers' inability to remain in their role drives up the cost of dementia care and increases its social burden such that even if dementia rates remained stable, health expenditures for dementia could come close to double the amount by 2040 because of the aging of the population [29]. Finding new and effective means of monitoring and managing the health and welfare of the caregiving population has obvious implications for controlling these costs.

Although the differences in cognitive performance between caregivers and controls were statistically significant, caregiver performance in our sample was still within 1 SD of the normed average. Thus, our caregivers' cognition was 'normal' relative to the general population average. However, because we had access to matched controls, we were able to detect differences in cognitive performance between caregivers and controls that may have been due to differences in psychosocial risk factors like stress and sleep. We speculate that these factors contribute in complex ways to caregivers' cognitive decline, a hypothesis that suggests the need for a better understanding of ways to assess and manage these aspects of caregiver burdens.

Chronic stress associated with caring for someone with dementia may explain some or all of the poorer cognitive performance observed in our caregiver sample [26]. Stress elevates cortisol levels in dementia caregivers [30], and high cortisol is linked to impaired cognitive functioning, particularly reduced attention and memory [31,32]. Stress also stimulates arousal and suppresses sleep $[33,34]$. We found that stress was generally a negative predictor of performance except at very low hours of sleep, at which point higher stress increased performance (Digit Symbol Coding), and at higher levels of education, which mediated the negative effect (Reverse Memory Span).

Although longer sleep generally increased cognitive performance for both Digit Symbol Coding and Forward Memory Span, it interacted in complex ways with stress and perceived support such that for high levels of stress, longer sleep decreased test performance. The impact of perceived social support was positive for Forward Memory Span; however, increased perceived social support had a negative effect on Reverse Memory Span perfor- 
mance. The latter finding contrasts with a previous study that linked increased social ties with favorable caregiver health outcomes [35].

It is important to note that although our regression analysis revealed statistically significant predictors of cognitive performance, none accounted for more than $7 \%$ of the variance in the data. One explanation for the modest performance of the models could be linked to the fact that the caregivers in this sample had cognitive profiles that were well within the normal range, and the distributions of many of their individual-level characteristics were also clustered. Our regression models, which focused only on caregivers, relied on outcome data that were clustered in a relatively small portion of the overall distribution of cognitive performance. Had our sample included larger numbers of caregivers with markedly poorer performance, or more detailed psychosocial measures, it is possible that the models would have explained more of the variability in the data.

Another explanation for the low model performance could be residual confounding by unmeasured factors such as depression [12, 27, 36-38]. It has been estimated that between 11 and $37 \%$ of caregivers suffer from depression [39-41], and previous studies have tied impaired sleep [10] and decreased social ties [35] to depression. Given these links, our findings suggest that future studies should target caregiver depression in parallel with other, related factors. In fact, caregiver interventions are most effective when they are comprehensive or multidimensional $[42,43]$ and targeted to meet the needs of individual caregivers [44]. Technological innovations that allow for virtual engagement are well suited to addressing this problem by bringing services to caregivers when and where they are needed.

This study has a number of important limitations. We did not have lifestyle data for controls, precluding an examination of these factors across the two study groups. The caregiver population in this study was self-identified and, by definition, consisted of individuals who have access to and were comfortable with computers and who had time to complete the assessments. However, all Web-based research targets individuals with computers, a consideration that will become less relevant over time as secular trends related to computer use in older age become more prevalent. An additional consideration concerns the fact that although most of the caregivers in our sample were informal, others were paid. The distinction between paid and unpaid caregivers is linked to age, with paid caregivers typically being younger than informal caregivers, who are often adult children of the care recipient. Although this heterogeneity may have impacted our results, it reflects the caregiving landscape and is therefore important to recognize and understand. Our results are also limited in that they are crosssectional and focused on cognitive testing at a single time point. Despite the cross-sectional nature of the data, our cohort of dementia caregivers represents one of the largest samples of its kind, and the fact that many of these individuals consented to future study indicates that we will be able to explore longitudinal relationships among the same individuals going forward. The study also did not include measures of attention, cognitive flexibility, or problem solving, key cognitive domains that contribute to performing complex functional activities, which in future studies could provide insight into the relationship to cognitive performance and activities of daily living. Finally, despite relatively low $\mathrm{R}^{2}$ values for our models, many of the relationships we observed indicated that even among the relatively healthy caregivers in our sample, there are measurable decrements in cognitive function. This suggests that caregivers with early decline may be identified with inexpensive screening technologies that, in turn, may facilitate provision of support that can help caregivers remain effective in their roles. 


\section{Acknowledgements}

AnthroTronix Inc. and Lumos Labs supported this research through internal research and development funds. The authors would like to thank the following partners for their outreach and scientific contributions to this study: the BrightFocus ${ }^{\circledR}$ Foundation, the Geoffrey Beene Foundation Alzheimer's Initiative, and Dr. Murali Doraiswamy of the Duke Institute for Brain Sciences, Durham, N.C., USA.

\section{Disclosure Statement}

The authors declare the following potential conflicts of interest with respect to the research, authorship, and/or publication of this article: Lumos Labs is the developer of the BPT.

\section{References}

1 Alzheimer's Association: 2015 Alzheimer's disease facts and figures. Alzheimers Dement 2015;11:332-384.

-2 Miller LS, Lewis MS, Williamson GM, Lance CE, Dooley WK, Schulz R, Weiner MF: Caregiver cognitive status and potentially harmful caregiver behavior. Aging Ment Health 2006;10:125-133.

-3 Chari AV, Engberg J, Ray K, Mehrotra A: The opportunity costs of informal elder-care in the United States: new estimates from the American Time Use Survey. Health Serv Res 2015;50:871-882.

$\checkmark 4$ Vitaliano PP, Echeverria D, Yi J, Phillips PEM, Young H, Siegler IC: Psychological mediators of caregiver stress and differential cognitive decline. Psychol Aging 2005;20:402-411.

5 Kannan H, Bolge SC, Del Valle M, Alvir J, Petrie CD: The association between Alzheimer's disease symptom severity and caregiver outcomes: a cross-sectional study. Prim Care Companion CNS Disord 2011; 13:PCC.10m01043.

6 Pinquart M, Sörensen S: Differences between caregivers and noncaregivers in psychological health and physical health: a meta-analysis. Psychol Aging 2003;18:250-267.

7 Mackenzie CS, Smith MC, Hasher L, Leach L, Behl P: Cognitive functioning under stress: evidence from informal caregivers of palliative patients. J Palliat Med 2007;10:749-758.

$>8$ Caswell LW, Vitaliano PP, Croyle KL, Scanlan JM, Zhang J, Daruwala A: Negative associations of chronic stress and cognitive performance in older adult spouse caregivers. Exp Aging Res 2003;29:303-318.

-9 Lee S, Kawachi I, Grodstein F: Does caregiving stress affect cognitive function in older women? J Nerv Ment Dis 2004;192:51-57.

$\rightarrow 10$ Oken BS, Fonareva I, Wahbeh H: Stress-related cognitive dysfunction in dementia caregivers. J Geriatr Psychiatry Neurol 2011;24:191-198.

11 Gaugler JE, Davey A, Pearlin LI, Zarit SH: Modeling caregiver adaptation over time: the longitudinal impact of behavior problems. Psychol Aging 2000;15:437-450.

12 Vitaliano PP, Zhang J, Young HM, Caswell LW, Scanlan JM, Echeverria D: Depressed mood mediates decline in cognitive processing speed in caregivers. Gerontologist 2009;49:12-22.

13 Corrêa MS, Vedovelli K, Giacobbo BL, de Souza CEB, Ferrari P, de Lima Argimon II, Walz JC, Kapczinski F, Bromberg E: Psychophysiological correlates of cognitive deficits in family caregivers of patients with Alzheimer disease. Neuroscience 2015;286:371-382.

14 Simone C, Ng N, Farzin F, Hardy JL, Scanlon MD: Performance on an online neuropsychological assessment is correlated with performance on standardized academic tests in middle school students (poster). Presented at the Neuroscience Conference, Washington, 2014.

15 Lawlor-Savage L, Goghari VM: Can a 15-minute online game replace Wechsler Test of Intelligence? Annu Meet Canadian Soc Brain Behav Cogn Sci, Calgary, 2013.

16 Morrison GE, Simone CM, Ng NF, Hardy JL: Reliability and validity of the NeuroCognitive Performance Test, a web-based neuropsychological assessment. Front Psychol 2015;6:1652.

-17 Sternberg DA, Ballard K, Hardy JL, Katz B, Doraiswamy PM, Scanlon M: The largest human cognitive performance dataset reveals insights into the effects of lifestyle factors and aging. Front Hum Neurosci 2013; 7:292.

18 Royer F: Information processing of visual figures in the Digit Symbol Substitution Task. J Exp Psychol 1971; 87:335-342.

19 Milner B: Interhemispheric differences in the localization of psychological processes in man. Br Med Bull 1971;27:272-277.

20 Psychological Corporation: Updated WAIS-III/WMS-III Technical Manual. San Antonio, Psychological Corporation, 2002. 
21 Pearson D, Sahraie A: Oculomotor control and the maintenance of spatially and temporally distributed events in visuo-spatial working memory. Q J Exp Psychol A 2003;56:1089-1111.

22 Iverson GL, Lange RT: Moderate and severe traumatic brain injury; in Schoenberg MR, Scott JG (eds): The Little Black Book of Neuropsychology: A Syndrome-Based Approach. New York, Springer, 2011, pp 663-696.

23 Army Individual Test Battery: manual of directions and scoring. Washington, War Department, Adjutant General's Office, 1944.

24 Reitan RM, Wolfson D: The Halstead-Reitan Neuropsychological Test Battery: Theory and Clinical Interpretation, ed 2. Tuscon, Neuropsychology Press, 1993.

25 Wechsler D: Manual for the Wechsler Adult Intelligence Scale. Oxford, Psychological Corp, 1955.

26 de Vugt ME, Jolles J, van Osch L, Stevens F, Aalten P, Lousberg R, Verhey FRJ: Cognitive functioning in spousal caregivers of dementia patients: findings from the prospective MAASBED study. Age Ageing 2006;35:160166.

27 Vitaliano PP, Murphy M, Young HM, Echeverria D, Borson S: Does caring for a spouse with dementia promote cognitive decline? A hypothesis and proposed mechanisms. J Am Geriatr Soc 2011;59:900-908.

28 Katzman R, Brown T, Fuld P, Peck A, Schechter R, Schimmel H: Validation of a short orientation-memoryconcentration test of cognitive impairment. Am J Psychiatry 1983;140:734-739.

29 Hurd MD, Martorell P, Delavande A, Mullen KJ, Langa KM: Monetary costs of dementia in the United States. N Engl J Med 2013;368:1326-1334.

-30 de Vugt ME, Nicolson NA, Aalten P, Lousberg R, Jolle J, Verhey FR: Behavioral problems in dementia patients and salivary cortisol patterns in caregivers. J Neuropsychiatry Clin Neurosci 2005;17:201-207.

-31 Lupien SJ, Lecours AR, Lussier I, Schwartz G, Nair NP, Meaney MJ: Basal cortisol levels and cognitive deficits in human aging. J Neurosci 1994;14:2893-2903.

-32 Lupien SJ, de Leon M, de Santi S, Convit A, Tarshish C, Nair NP, Thakur M, McEwen BS, Hauger RL, Meaney MJ: Cortisol levels during human aging predict hippocampal atrophy and memory deficits. Nat Neurosci 1998;1: 69-73.

33 Chrousos GP: Stress and disorders of the stress system. Nat Rev Endocrinol 2009;5:374-381.

-34 Chrousos GP: Organization and integration of the endocrine system. Sleep Med Clin 2007;2:125-145.

-35 Cannuscio CC, Colditz GA, Rimm EB, Berkman LF, Jones CP, Kawachi I: Employment status, social ties, and caregivers' mental health. Soc Sci Med 2004;58:1247-1256.

-36 Roca M, Vives M, López-Navarro E, García-Campayo J, Gili M: Cognitive impairment and depression: a critical review. Actas Esp Psiquiatr 2015;43:187-193.

37 Harvey PD: Cognitive impairments in major depression and bipolar disorders. Psychiatry 2007;4:12-14.

-38 Spira JL, Lathan CE, Bleiberg J, Tsao JW: The impact of multiple concussions on emotional distress, postconcussive symptoms, and neurocognitive functioning in active duty United States marines independent of combat exposure or emotional distress. J Neurotrauma 2014;31:1823-1834.

39 Covinsky KE, Newcomer R, Fox P, Wood J, Sands L, Dane K, Yaffe K: Patient and caregiver characteristics associated with depression in caregivers of patients with dementia. J Gen Intern Med 2003;18:1006-1014.

40 Mahoney R, Regan C, Katona C, Livingston G: Anxiety and depression in family caregivers of people with Alzheimer disease: the LASER-AD study. Am J Geriatr Psychiatry 2005;13:795-801.

-41 Joling KJ, van Marwijk HWJ, Veldhuijzen AE, van der Horst HE, Scheltens P, Smit F, van Hout HPJ: The two-year incidence of depression and anxiety disorders in spousal caregivers of persons with dementia: who is at the greatest risk? Am J Geriatr Psychiatry 2015;23:293-303.

42 Belle SH, Burgio L, Burns R, Coon D, Czaja SJ, Gallagher-Thompson D, et al: Enhancing the quality of life of dementia caregivers from different ethnic or racial groups: a randomized, controlled trial. Ann Intern Med 2006;145:727-738.

43 Drentea P, Clay OJ, Roth DL, Mittelman MS: Predictors of improvement in social support: five-year effects of a structured intervention for caregivers of spouses with Alzheimer's disease. Soc Sci Med 2006;63:957-967.

-44 Montgomery RJV, Kwak J, Kosloski K, O'Connell Valuch K: Effects of the TCARE ${ }^{\circledR}$ intervention on caregiver burden and depressive symptoms: preliminary findings form a randomized controlled study. J Gerontol B Psychol Sci Soc Sci 2011;66:640-647. 